\title{
Acute effects of therapeutic irradiation for prostatic carcinoma on anorectal function
}

\author{
E K Yeoh, A Russo, R Botten, R Fraser, D Roos, M Penniment, M Borg, W M Sun
}

\begin{abstract}
Aim-The incidence of anorectal symptoms after radiotherapy (RTH) for localised pelvic malignant disease is unclear. In addition, the effects of pelvic irradiation on both anorectal motility and sensory function are poorly defined. A prospective study was therefore performed on 35 patients (55-82 years of age) with localised prostatic carcinoma before and four to six weeks after RTH to assess its effects on anorectal function.

Methods-Anorectal symptoms were assessed by questionnaire. Anorectal pressures at rest and in response to voluntary squeeze, rectal distension, and increases in intra-abdominal pressure were evaluated with perfused sleeve side hole manometry. Rectal sensation was tested during graded balloon distension. Rectal compliance was calculated by the pressure-volume relation obtained during the testing of rectal sensation. Ultrasound was used to determine anal sphincter structure and integrity.
\end{abstract}

Results-RTH had no effect on anal sphincter morphology. The frequency of defecation increased after RTH (7 (3-21) $v$ 10 (3-56) bowel actions a week; $p<0.01$ ). After RTH, 16 patients had faecal urgency and eight faecal incontinence, compared with five and one respectively before RTH ( $p<0.01$ for each). Basal and squeeze sleeve recorded pressures were reduced after RTH (54 (3) $v 49$ (3) $\mathrm{mm} \mathrm{Hg}(\mathrm{p}<0.05)$ and $111(8) v 102(8) \mathrm{mm} \mathrm{Hg}(\mathrm{p}<0.01)$, before and after RTH respectively; means (SEM)). Rectal compliance was reduced after RTH (1.2 $v 1.4 \mathrm{~mm} \mathrm{Hg} / \mathrm{ml}, \mathrm{p}<0.05)$. After RTH, threshold volumes for perception of rectal distension were lower in the 16 patients who either experienced faecal urgency for the first time (13 patients) or reported worsening of this symptom (three patients) compared with the remaining patients (34 (4) $v 48$ (5) $\mathrm{ml}$ respectively, p<0.05).

Conclusion-Faecal incontinence (23\%) is a common problem four to six weeks after RTH for prostatic carcinoma and is associated with minor reductions in anal sphincter pressures. The high prevalence of faecal urgency in patients after RTH may be related to alterations in rectal perception of stool.

(Gut 1998;43:123-127)

Keywords: anorectal function; radiotherapy; motility; manometry; incontinence
The incidence of prostatic carcinoma has increased notably in recent years. ${ }^{1}$ This can be attributed to a number of factors, including the increasing life expectancy of men and greater public awareness of prostate cancer. In addition, the more frequent use of serum prostate specific antigen, transrectal ultrasound, and prostate biopsies has resulted in the detection of asymptomatic disease. ${ }^{2}$ Although many asymptomatic prostate cancers do not cause clinically significant disease within the lifetime of the patient, ${ }^{3}$ it is not yet possible to predict the likelihood of progression in individual patients and there is a lack of consensus as to optimal treatment.

Primary radiation therapy $(\mathrm{RTH})$ has been the standard treatment for early stage disease. ${ }^{4}$ Although radical prostatectomy has been used as an alternative treatment, its use is currently limited to younger men with organ confined disease and without co-morbidities that increase operative risks. ${ }^{5}$ There is a high incidence of urogenital complications such as urinary incontinence and sexual impotence after radical prostatectomy. ${ }^{6}$ Therefore, the demand for RTH for the treatment of prostatic carcinoma is likely to continue increasing, particularly since 10 year disease specific survival is comparable with surgical resection with a lower incidence of long term complications. ${ }^{7}$ It is generally acknowledged that a degree of acute radiation proctitis characterised by increased frequency and urgency of defecation, rectal pain, and bleeding is common during RTH. ${ }^{89}$ In about $10 \%$ of patients undergoing pelvic irradiation for urogenital malignant disease, acute radiation proctitis is severe enough to interrupt the scheduled plan of treatment and reduces cure rates. ${ }^{9-11}$

The pathophysiology and natural history of acute radiation proctitis is poorly understood. Although the morphological effects of pelvic irradiation on rectal mucosa are well documented, ${ }^{912}$ only one study has evaluated the effects of pelvic irradiation on anorectal motor function. ${ }^{13}$ Data from this study suggested that there were no changes in anorectal motility; however, the anal sphincter was in the field of radiation in only half the patients. ${ }^{13}$

We therefore performed a prospective study of anorectal function and anal sphincter structure before and four to six weeks after irradiation. The aim of the study was to evaluate the short term effects of pelvic irradiation for the curative treatment of prostatic carcinoma on anorectal function.

Materials and methods

SUBJECTS

A total of 35 male patients (median age 68 (range 55-82), median weight 76 (range 55-115) kg, median body mass index 26 (range
Accepted for publication 4 February 1998 
20-38) $\mathrm{kg} / \mathrm{m}^{2}$ ) with localised (confined to the organ) prostatic carcinoma were recruited from patients referred to four radiation oncologists (EKY, DR, MP, and MB) at the Royal Adelaide Hospital between July 1996 and May 1997. Patients with a serum prostatic specific antigen level greater than $80 \mathrm{mg} / \mathrm{ml}$ were excluded, as were those who had received antiandrogen therapy. Patients with an ongoing need for medication likely to influence anorectal motility such as antidiarrhoeal medication-for example, loperamide - and stimulant laxatives-for example, coloxyl with senna-were also excluded. Eligible patients were treated with one of two radiation dose regimens, $55 \mathrm{~Gy} / 20$ fractions/four weeks (18 patients) or $64 \mathrm{~Gy} / 32$ fractions/ 6.5 weeks (17 patients). The radiation dose was prescribed to the isocentre (point of intersection of the radiation beams in the centre of the irradiated tumour volume).

PROTOCOL

Each patient was evaluated by one of two investigators ( $\mathrm{RB}$ or $\mathrm{AR}$ ) for the following aspects of anorectal function both before and four to six weeks after completion of RTH: (a) symptoms, (b) anorectal motility and sensation, and (c) anal ultrasound. Written informed consent was obtained from all patients and the study protocol was approved by the human ethics committee of the Royal Adelaide Hospital.

\section{Anorectal symptoms}

The following symptoms were assessed by questionnaire: bowel frequency (number of bowel actions a week), urgency of defecation, and faecal incontinence (diurnal and nocturnal).

The scores for both urgency of defecation and faecal incontinence (diurnal and nocturnal) were based on ( $a$ ) frequency and $(b)$ severity of each of the two symptoms. For urgency of defecation, frequency was scored as follows: 0 $=$ less than one episode a week; $1=$ less than three episodes a week; $2=$ three or more episodes a week; 3 = one episode a day. Severity was scored as: $0=$ absence of symptoms; 1 $=$ mild, symptoms could be ignored; $2=$ moderate, symptoms could not be ignored, but did not influence daily activities; 3 = severe, symptoms influenced daily activities. A maximum score of 6 was obtainable. ${ }^{14}$

The criteria for scoring frequency of faecal incontinence was identical with that for urgency of defecation (see above), although diurnal and nocturnal frequency of faecal incontinence were scored separately. The scoring of severity of faecal incontinence (both diurnal and nocturnal) was based on three grades of severity as in a previous study, ${ }^{15}$ but modified to take into account the need for incontinence pads according to the following classification: $0=$ absence of faecal incontinence; $1=$ predominantly incontinent of flatus; $2=$ incontinence necessitating the wearing of a pad; $3=$ incontinence necessitating a change of pad more than once a day. ${ }^{16}$ The total score for faecal incontinence was therefore 12 , and the grand total score of anorectal symptoms (maximum 18) was also calculated for each patient. ${ }^{16}$
Anorectal manometry

Each patient was instructed to empty the rectum before the study and digital examination was performed to ensure that the rectum was empty. Patients lay in the left lateral position with the hips flexed at $90^{\circ}$. The manometric assembly was introduced along the posterior wall of the anorectum. The manometric assembly (outer diameter $4.4 \mathrm{~mm}$ ) incorporated side holes located at $1,2,3,4,8$, and 20 $\mathrm{cm}$ from the anal verge, a sleeve sensor, and a $5.5 \mathrm{~cm}$ silicone rubber balloon. The latter was located between 10.5 and $16 \mathrm{~cm}$ from the anal verge. The sleeve sensor, which was $4 \mathrm{~cm}$ in length, straddled the anal canal with one end just proximal to the anal verge. The sleeve sensor records the highest pressure along the anal canal irrespective of its position. All channels, except the balloon channel, were perfused with degassed distilled water at a rate of $0.6 \mathrm{ml} / \mathrm{min}$ by a pneumohydraulic pump. The balloon channel was water filled but non-perfused. The pressure measurements were digitised at $10 \mathrm{~Hz}$ on line and stored on a Macintosh Quadra 700 computer for later analysis.

Anorectal pressures were initially recorded for 10 minutes under resting conditions to ensure that steady state pressures were measured. The patient was then instructed to contract the anal sphincter maximally (squeeze) for 15 seconds on three separate occasions one minute apart. After a further 10 minute rest period, the rectal balloon was inflated with $10,20,40,60,100$, and $150 \mathrm{ml}$ of air (the patient was unaware of the nature, order, or grade of the stimulus). Each inflation was maintained for one minute, and, after deflation of the balloon, at least one minute was allowed before the next inflation. During each inflation the patient was asked to report the occurrence and nature of rectal sensationthat is, perception of the balloon, gas (wind), desire to defecate, discomfort, or pain. Distension was terminated if the subject felt discomfort or pain at any volume or at a maximum volume of $150 \mathrm{ml}$. A further 10 minute rest period followed. To increase intra-abdominal pressure, each patient blew up a $23 \mathrm{~cm}$ diameter party balloon (Pioneer Ansell Worldwide, Brookvale, NSW, Australia) three times, separated by one minute.

\section{Anal ultrasound}

At the end of the manometric study, the catheter was removed and a $7.5 \mathrm{MHz}$ rotating ultrasonographic scanner (Bruel and Kjaer, Naerum, Denmark) with an outer diameter of $1 \mathrm{~cm}$ was positioned just proximal to the anal margin. Cross sectional images were obtained at eight equidistant points in the anal canal. The maximal thickness of both internal anal sphincter (IAS) and external anal sphincter (EAS) was determined and the mean thickness of the IAS and EAS calculated. ${ }^{17}$

\section{DATA ANALYSIS}

The following parameters of anorectal motility were determined using semi-automated analysis by AcqKnowledge (Version 3.2; BIOPAC Systems, Santa Barbara, California, USA): (a) 
Table 1 Number of patients with anorectal symptoms before and after radiotherapy (RTH)

\begin{tabular}{llllll}
\hline \multirow{2}{*}{ Treatment } & \multicolumn{2}{l}{ Faecal incontinence } & & \\
\cline { 2 - 4 } & Diurnal & Nocturnal & Both & Faecal incontinence & Faecal urgency \\
\hline Before RTH & $1 / 35$ & $0 / 35$ & $0 / 35$ & $1 / 35$ & $5 / 35$ \\
After RTH & $6 / 35$ & $0 / 35$ & $2 / 35$ & $8 / 35^{\star \star}$ & $16 / 35^{\star \star}$ \\
\hline
\end{tabular}

${ }^{\star \star} \mathrm{p}<0.01 v$ before RTH.

minimum basal pressure, defined as the lowest mean pressure recorded by the sleeve that was sustained for more than two minutes during the baseline period; $(b)$ the plateau pressure during sustained squeeze recorded by the sleeve sensor; (c) the plateau anal pressure when the subject was blowing up a party balloon, measured using the sleeve sensor; $(d)$ the pressure profile curves, including all anal channels; (e) threshold volumes at which rectal balloon distension was first perceived; $(f)$ the pressurevolume relation for rectal balloon distension. Rectal compliance was then calculated from the maximal slope between 40 and $100 \mathrm{ml} .{ }^{18}$

\section{STATISTICAL ANALYSIS}

Symptom scores obtained before and after RTH were analysed using a $\chi^{2}$ test. Frequency of defecation, rectal compliance, and anal ultrasound data obtained before and after RTH were compared using the Wilcoxon rank sum test. Pressures from the sleeve sensor and threshold volumes of rectal sensation were analysed using Student's $t$ test. Manometric pressure profiles of anal channels were analysed using analysis of variance. Subgroup analysis was performed accordingly using both the Mann-Whitney $\mathrm{U}$ and unpaired $t$ test. Relations between anorectal symptom scores, bowel actions per week, basal pressures, and pressures generated in response to voluntary squeeze and rectal distension were evaluated using linear regression analysis. $p<0.05$ was considered to be significant in all analyses.

\section{Results}

ANORECTAL SYMPTOMS

The scores for urgency of defecation and faecal incontinence were greater after RTH than before $(1.4(0.3) v 0.4(0.2)$ and $1(0.4) v 0.09$ (0.09) respectively; $\mathrm{p}<0.01$ for both). The total symptom score was greater after RTH than before $(2.4 \quad(0.6) \quad v \quad 0.5 \quad(0.2)$ respectively; $\mathrm{p}<0.01)$. Mean frequency of defecation was 10 (3-56) bowel actions a week after RTH compared with $7(3-21)$ before $(\mathrm{p}<0.01)$. Stool consistency was unaffected by RTH: before RTH, 6/35 patients had hard stool, 26/35 had

Table 2 Maximum thickness of the internal (IAS) and external (EAS) anal sphincters, anal pressures (sleeve), rectal compliance, and rectal sensation in patients before and after radiotherapy $(\mathrm{RTH})$

\begin{tabular}{llll}
\hline & Before $R T H$ & After RTH & p Value \\
\hline EAS $(\mathrm{mm})^{\star}$ & $9.4(5.8-18.0)$ & $10(6.2-17.0)$ & $\mathrm{NS}$ \\
IAS $(\mathrm{mm})^{\star}$ & $2.4(1.1-4.2)$ & $2.2(1.5-4.0)$ & $\mathrm{NS}$ \\
Basal pressure $(\mathrm{mm} \mathrm{Hg}) \dagger$ & $54(3)$ & $49(3)$ & 0.05 \\
Voluntary squeeze pressure $(\mathrm{mm} \mathrm{Hg}) \dagger$ & $111(8)$ & $102(8)$ & 0.008 \\
Blow up a party balloon $(\mathrm{mm} \mathrm{Hg}) \dagger$ & $95(5)$ & $92(6)$ & $\mathrm{NS}$ \\
Rectal compliance $(\mathrm{mm} \mathrm{Hg} / \mathrm{ml}) \dagger$ & $1.2(0.06)$ & $1.4(0.08)$ & 0.05 \\
Rectal sensation $(\mathrm{ml}) \dagger$ & $36(4)$ & $42(4)$ & $\mathrm{NS}$ \\
\hline
\end{tabular}

Data expressed as ${ }^{\star}$ median (range) or tmean (SEM).
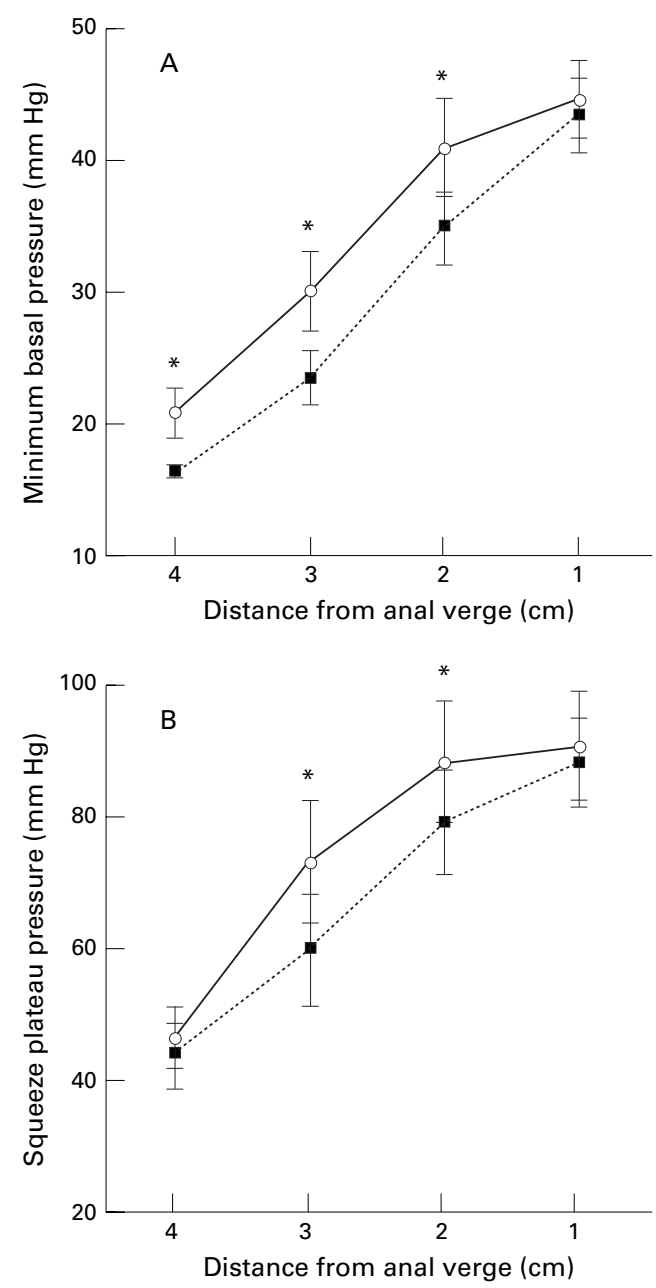

Figure 1 Pressure along the anal canal. Changes in minimum basal $(A)$ and squeeze plateau $(B)$ pressure in patients before (open circles) and after (closed squares) radiotherapy. Values are mean $(S E M) .{ }^{\star} p<0.05 v$ after radiotherapy.

soft stool, 3/35 had loose (unformed) stool; after RTH, 9/35 had hard stool, 22/35 had soft stool, $4 / 35$ had loose (unformed) stool $(p=0.7)$.

Before RTH, five patients had mild to moderate faecal urgency (score 2-4 out of maximum of 6) and one had mild faecal incontinence (score 3 out of maximum of 12) (table 1). Sixteen patients (46\%) either developed faecal urgency for the first time (13 patients) after RTH or reported a worsening of preexisting urgency of defecation (three patients) $(\mathrm{p}<0.01)$ (table 1). Seven patients $(20 \%)$ developed faecal incontinence after RTH $(\mathrm{p}<0.01)$ (table 1). The one patient who had mild faecal incontinence before RTH reported no worsening of this symptom after RTH. Eight $(23 \%)$ patients experienced both faecal urgency and incontinence. Thirteen patients (37\%) experienced an increase in bowel frequency.

There was an association between total symptom score and the number of bowel actions a week after RTH $(r=0.536$, p<0.001). Similarly, there was a relation between the number of bowel actions a week and faecal incontinence after RTH $(r=0.421, \mathrm{p}<0.01)$, as well as urgency of defecation $(r=0.483$, $\mathrm{p}<0.01$ ). 
Table 3 Anorectal symptoms, pressures, rectal compliance, and rectal sensation in patients with and without symptoms after radiotherapy

\begin{tabular}{|c|c|c|c|c|c|c|c|c|c|}
\hline \multirow[b]{2}{*}{ Patient subgroup } & \multirow[b]{2}{*}{ No } & \multicolumn{3}{|l|}{ Symptom score } & \multirow[b]{2}{*}{$\begin{array}{l}\text { No of bowel } \\
\text { actions/week } \neq\end{array}$} & \multirow{2}{*}{$\begin{array}{l}\text { Basal } \\
\text { pressure } \\
(m m \mathrm{Hg}) \dagger\end{array}$} & \multirow{2}{*}{$\begin{array}{l}\text { Squeeze } \\
\text { pressure } \\
(m m \mathrm{Hg}) \dagger\end{array}$} & \multirow{2}{*}{$\begin{array}{l}\text { Rectal } \\
\text { compliance } \\
(m m \mathrm{Hg} / \mathrm{ml}) \neq\end{array}$} & \multirow[b]{2}{*}{$\begin{array}{l}\text { Rectal sensation } \\
(\mathrm{ml}) t\end{array}$} \\
\hline & & $\begin{array}{l}\text { Faecal urgency } \\
\text { score } \neq\end{array}$ & $\begin{array}{l}\text { Faecal incontinence } \\
\text { score } \neq\end{array}$ & Total score ${ }^{\star}$ & & & & & \\
\hline With urgency & $16 / 35$ & - & $1(0-10)^{\star \star}$ & $4(1-15)^{\star \star}$ & $12(3-56)$ & $48(4)$ & $95(8)$ & $1.5(0.5-2.2)$ & $34(4)^{\star}$ \\
\hline Without urgency & $19 / 35$ & - & $0(0-0)$ & $0(0-3)$ & $7(7-18)$ & $47(5)$ & $108(14)$ & $1.4(0.3-2.2)$ & $48(5)$ \\
\hline With faecal incontinence & $7 / 35$ & $2(1-5)^{\star}$ & - & $6(4-15)^{\star \star}$ & $11(7-56)$ & $48(7)$ & $99(13)$ & $1.6(1.4-1.9)$ & $33(6)$ \\
\hline Without faecal incontinence & $28 / 35$ & $0(0-6)$ & - & $0(0-6)$ & $9(3-28)$ & $49(3)$ & $102(10)$ & $1.4(0.3-2.2)$ & $44(4)$ \\
\hline
\end{tabular}

Data expressed as $\neq$ median (range) or tmean (SEM).

${ }^{\star} \mathrm{p}<0.05 v$ without symptoms.

$\star \star \mathrm{p}<0.01 v$ without symptoms.

ANORECTAL MANOMETRY

The pressures in the anal canal showed a positive gradient towards the anal verge for both minimum basal and squeeze plateau pressures before and after RTH. Pressures in the anal canal were lower after RTH than before (fig 1). Minimum basal pressure measured by the sleeve sensor was lower after RTH than before $(\mathrm{p}<0.05$, table 2$)$. Similarly, mean squeeze pressure recorded by the sleeve was also reduced after $\mathrm{RTH}(\mathrm{p}<0.01)$ (table 2$)$. In contrast, anal pressures in response to blowing up a balloon after RTH did not differ from those recorded before RTH (table 2).

Rectal balloon distension was perceived by all subjects. The threshold for perception of rectal balloon distension was not changed significantly after $\mathrm{RTH}$, despite a decrease in rectal compliance $(\mathrm{p}<0.01)$ (table 2$)$.

\section{ANORECTAL ULTRASOUND}

RTH had no effect on the thickness of either IAS or EAS (table 2).

\section{ANORECTAL DATA IN PATIENTS WITH FAECAL URGENCY}

The 16 patients who either experienced faecal urgency or reported a worsening of the pre-existing symptom after RTH had a higher faecal incontinence score $(1(0-10))$ than the remaining 19 patients $(0(0-0), \mathrm{p}<0.01)$ (table 3). Before RTH, patients who subsequently developed faecal urgency perceived rectal distension at a lower volume than patients who did not develop urgency (28 (5) $v 42$ (5) $\mathrm{ml}, \mathrm{p}$ $=0.05)$. After RTH, the volume at which rectal balloon distension was perceived remained lower in the 16 patients who experienced faecal urgency or reported a worsening of this symptom compared with the remaining 19 patients (34 (4) v 48 (5) $\mathrm{ml}, \mathrm{p}<0.05)$. There was, however, a slight increase in volumes (about 10\%) in both groups of patients after RTH but no difference in rectal compliance (table 3 ).

ANORECTAL DATA IN PATIENTS WITH FAECAL INCONTINENCE

Data were analysed separately in seven patients who experienced faecal incontinence after RTH. These patients had a higher score for urgency of defecation $2(1-5)$ than the remaining 28 patients $(0(0-6), \mathrm{p}<0.01)$ (table 3$)$. There were no significant differences in anorectal pressures or rectal sensory perception between these two subgroups of patients (table 3).

\section{Discussion}

The major finding in this study is that four to six weeks after pelvic irradiation faecal urgency developed or worsened in $16(46 \%)$ of 35 patients, seven of whom $(44 \%)(20 \%$ of the whole group) were also incontinent of faeces. These symptoms were associated with alterations in anorectal function in the absence of obvious morphological changes in the anal sphincters. Both the minimum basal and mean squeeze pressures were reduced after RTH, suggesting dysfunction of both internal and external anal sphincters. In addition, rectal compliance was reduced after RTH, although the threshold perception of rectal distension was not significantly changed. Although the magnitude of the changes in individual anorectal parameters after RTH in the current study were relatively small, they may nevertheless be clinically significant, especially if the mechanisms that usually maintain continence are stressed.

This study confirms the high frequency of anorectal symptoms after pelvic irradiation for urological malignant disease reported previously. ${ }^{9}$ The prevalence of faecal urgency after RTH is almost identical with the $44 \%$ reported by Sedgwick et al. ${ }^{9}$ Although the prevalence of faecal incontinence four weeks after RTH was not reported separately from faecal urgency, ${ }^{9}$ incontinence was noted in about $30 \%$ of patients during RTH. In the current study, only $20 \%$ of patients were incontinent of faeces at four to six weeks after RTH. The lower prevalence probably reflects a degree of recovery of anorectal function after RTH. Other differences may relate to the patient population and radiation dose prescribed.

It has previously been reported that there is no correlation between sigmoidoscopic and histological changes in rectal mucosa and severity of symptoms. ${ }^{9}$ Thus, although it is possible that mucosal inflammatory changes are responsible for the persistence of anorectal symptoms, it is likely that other mechanisms such as neural and muscular injury also contribute to the development of symptoms such as faecal urgency and incontinence. Our group $^{16}$ and others ${ }^{19}{ }^{20}$ have shown that late changes, such as anorectal symptoms associated with reduced minimal basal pressures and pressures in response to squeeze, decreased rectal compliance and a consequent increase in rectal sensitivity, are common a number of years after pelvic irradiation for gynaecological and urological malignant disease. We previ- 
ously suggested that myogenic rather than neurogenic mechanisms are probably involved in the anorectal motility disturbances, as anal sphincter electromyography was unchanged. ${ }^{16}$ Although concurrent electromyography of the anal sphincters was not performed in the current study, it is likely that similar mechanisms account for the anal sphincter dysfunction. However, the underlying pathogenesis of reduced rectal compliance in the current study is probably not fibrosis, as this is not usually seen for at least two months after RTH. ${ }^{21}$ We speculate that these changes may result from submucosal oedema.

Birnbaum et $a l^{13}$ suggested that RTH does not lead to changes in anorectal motor function. However, in that study patients with rectal carcinoma were only evaluated before surgery. The anal canal was not in the radiation field in half of the patients as opposed to the current study where radiation of the prostatic bed included the anal canal in all patients (as shown from computed tomography planning scans). In addition, the patients in the previous study ${ }^{13}$ received a total radiation dose that was $60-70 \%$ of that in the current study, which may have been insufficient to result in neuromuscular injury. Differences in anorectal manometric technique may also have contributed to the discrepancies. In the earlier study, a station withdrawal technique was used to measure anorectal pressure, whereas in the current study multiport manometry incorporating a sleeve sensor at the anal canal was utilised. Unlike station withdrawal manometry, the multiport technique facilitates the interpretation of manometric profiles. In addition, the sleeve sensor allows maximum pressures to be recorded because of a greater tolerance of axial movement. ${ }^{22}$

Our data show that four weeks after RTH, the threshold of perception to rectal distension was lower in patients who experienced urgency of defecation or faecal incontinence than in patients without these symptoms, despite similar anal sphincter pressures. It is of note that patients who developed urgency had lower volumes for rectal sensory perception both before and after RTH, although RTH per se did not affect the volumes at which rectal distension was perceived. These data are consistent with the changes seen in compliance after RTH. Further studies are needed to investigate the mechanisms that underlie this observation. We have previously shown that RTH for gynaecological malignancies has long term effects on anorectal function. ${ }^{16}$ The current study was designed to assess the acute effects of localised RTH on anorectal function for carcinoma of the prostate. It appears likely that chronic changes in anorectal motor and sensory function may also occur in this setting. Studies are therefore required to address this issue, together with the relation between acute and chronic effects of RTH on anorectal function.

Management of acute radiation anorectal sequelae is unsatisfactory in part because the pathophysiology of motor disturbances is unknown. Loperamide is frequently used to alleviate anorectal symptoms in patients with diarrhoea and faecal incontinence. This drug increases squeeze as well as basal anal sphincter pressures, ${ }^{23}$ slows down intestinal transit, and reduces stool volume. ${ }^{24}$ It is unclear which of these actions is most important. In addition, a reduction in stool volume could theoretically increase the risk of rectal bleeding from acutely inflamed rectal mucosa, even though it may reduce urgency and frequency of defecation by reducing stool bulk. A better understanding of the pathophysiology of anorectal motor and sensory function in acute radiation proctitis would allow targeted treatment.

Part of this study was presented in abstract form at the American Gastroenterology Association Meeting in Washington DC, May 1997.

1 Wingo PA, Tong T, Bolden S. Cancer statistics, 1995. $C A$ Cancer f Clin 1995;45:8-30.

2 Whitmore Jr WF. Localised prostatic cancer: management and detection issues. Lancet 1994;343:1263-7.

3 Carter HB, Piantadosis S, Issacs JT. Clinical evidence for and implications of the multi-step development of prostate cancer. F Urol 1990;143:742-6.

4 Hanks GE, Liebel SA, Krall JM, et al. Patterns of care studies: dose-response observations for local control of adenocarcinoma of the prostate. Int $\mathcal{F}$ Radiat Oncol Biol Phys 1995;11:153-7.

5 Morton RA, Steiner MS, Walsh PC. Cancer control following anatomical radical prostatectomy: an interim report. $\mathcal{F}$ Urol 1991;145:1197-200

6 Wasson JH, Cushman CC, Bruskewitz RC, et al. A structured literature review of treatment for localized prostate cancer. Arch Fam Med 1993;2:487-93.

7 Bagshaw MA, Cox RS, Hancock SL. Control of prostate cancer with radiotherapy: long-term results. $\mathcal{f}$ Urol 1994;152:1781-5.

8 Kearsley JH. High-dose radiotherapy for localized prostatic cancer. An analysis of treatment results and early complications. Med f Aust 1986;144:624-8.

9 Sedgwick DM, Howard GCW, Ferguson A. Pathogenesis of acute radiation injury to the rectum: a prospective study in patients. Int $\mathcal{F}$ Colorectal Dis 1994;9:23-30.

10 Amdur RJ, Parsons JT, Fitzgerald LT, et al. The effect of overall treatment time on local control in adenocarcinoma of prostate treated with radiation therapy. Int $\mathcal{F}$ Radiat Oncol Biol Phys 1990;19:1377-82.

11 Fyles A, Keane TJ, Barton M, et al. The effect of treatment duration in the local control of cervix cancer. Radiother Oncol 1992;25:273-9.

12 Gelfand MD, Tepper M, Katz LA, et al. Acute irradiation proctitis in man: development of eosinophilic crypt abscesses. Gastroenterology 1968;54: 401-11.

13 Birnbaum EH, Dreznik Z, Myerson RJ, et al. Early effect of external beam radiation therapy on the anal sphincter: a study using anal manometry and transrectal ultrasound. Dis Colon Rectum 1992;35:757-67.

14 Horowitz M, Harding PE, Chatterton BE, et al. Acute and chronic effects of domperidone on gastric emptying in diabetic autonomic neuropathy. Dig Dis Sci 1985;30:1-9.

15 Pescatori M, Anastasio G, Bottini C, et al. New grading and scoring for anal incontinence: evaluation of 335 patients. Dis Colon Rectum 1992;35:482-7.

16 Yeoh EK, Sun WM, Russo A, et al. A retrospective study of the effects of pelvic irradiation for gynaecological cancer on anorectal function. Int $\mathcal{F}$ Radiat Oncol Biol Phys 1996;35: 1003-10.

17 Sun WM, Peck J, Shorthouse AJ, et al. Haemorrhoids are associated not with hypertrophy of the internal anal sphincter but with hypertension of the anal cushions. $\mathrm{Br} \mathcal{F}$ Surg 1992;79:592-4.

18 Sun WM, Read NW, Prior A, et al. Sensory and motor response to rectal distension vary according to rate and response to rectal distension vary according to rate and
pattern of balloon inflation. Gastroenterolgy 1990;99:1008patter.

19 Varma JS, Smith AN, Busuttil A. Correlation of clinical and manometric abnormalities of rectal function following chronic radiation injury. Br F Surg 1985;72:875-8.

20 Varma JS, Smith AN, Busuttil A. Function of the anal sphincters after chronic radiation injury. Gut 1986;27:52833.

21 Rubin P, Casarett GW. Alimentary tract: small and large intestines and rectum. In: Rubin P, Casarett GW, eds. Clinical radiation pathology. Philadelphia: Saunders, 1968;1: 193-240.

22 Orkin BA, Hanson RB, Kelly KA, et al. Human anal motility while fasting, after feeding, and during sleep. Gastroenterology 1991;100:1016-23.

23 Read M, Read NW, Barber DC, et al. Effects of loperamide on anal sphincter function in patients complaining of chronic diarrhea with fecal incontinence and urgency. Dig Dis Sci 1982;27:807-14.

24 Yeoh EK, Horowitz M, Russo A, et al. Gastrointestinal function in chronic radiation enteritis: effects of function in chronic radiation enteritis:
loperamide-N-oxide. Gut 1993;34:476-82. 